\title{
THE EFFECT OF NANO-HYBRID COMPOSITE RESIN DENTURE TEETH ON MANDIBULAR RESIDUAL RIDGE
}

\author{
Maha Ahmed Hossny Moussa ${ }^{1}$, Moataz Mostafa Bahgat ElMahdy ${ }^{2}$ Sayed Mohamed Mohamed \\ El Masry ${ }^{3}$, Hala Mohamed Abdel Hameed Ali ${ }^{4}$
}

DOI: $10.21608 /$ dsu.2021.40818.1044

Manuscript ID: DSU-2008-1044 (R1)

\section{KEYWORDS}

Cone beam computed

tomography, nanocomposite

denture teeth, ridge resorption

- E-mail address: maha.mosa1990.mm@gmail.com

1. Demonstrator in Prosthodontic Department, Faculty of Dentistry, Suez Canal University

2. Professor of Prosthodontic Department, Faculty of Dentistry, Suez Canal University

3. Professor of Prosthodontic department, Faculty of Dentistry, Suez Canal University

4. Assistant Prof.of Prosthodontic department, Faculty of Dentistry, Suez Canal University

\begin{abstract}
Aim: This study was conducted to evaluate radiographically the effect of nano-hybrid composite resin teeth on mandibular residual ridge using cone beam computerized tomography ( $\mathrm{CBCT})$. A comparative study. Materials and Methods: Twelve completely edentulous male patients were randomly divided into two groups according to the artificial tooth material used in complete denture construction. One group received complete dentures with nano-hybrid composite resin teeth and the other group received complete dentures with cross-linked acrylic resin teeth. CBCT was taken at the delivery, 6 months and 12 months follow -up appointments. The evaluation was done for height and width changes of the mandibular residual alveolar ridge at different anatomical sites either in the anterior or posterior part of the mandible. Results: Analysis was done by using two-tailed t-test revealed that increase in amount of bone loss in both groups which is in favor to patients wearing dentures with nanohybrid composite resin teeth, with no statistically significant difference between the two groups. Conclusion: Despite of introduction of new denture tooth material as nanohybrid composite resin teeth, the cross-linked acrylic resin teeth still considered the gold standard denture teeth regarding the degree of occlusal force transmission and preservation of residual alveolar ridge.
\end{abstract}

\section{INTRODUCTION}

Progressive ridge resorption is one of the main causes of loss of stability and retention of mandibular complete dentures with subsequent loss of masticatory efficiency and patient discomfort ${ }^{(1)}$.

The transmission of masticatory forces to the underlying ridge may be influenced by internal structure and material of artificial teeth incorporated in the complete denture. In this context of transmission of forces to the residual ridge, the question arises of whether a material with a higher coefficient of elasticity, such as that used in acrylic resin artificial teeth, would be less harmful to the residual ridges ${ }^{(2,3)}$.

Traditional resin denture teeth have two shortcomings: 1. Conventional resins do not replicate light. 2. Inferior wear resistance of acrylic resin artificial teeth is a significant limitation for complete denture therapy ${ }^{(4)}$. 
The introduction of nano-hybrid composite denture teeth represented true innovation in the dental market. This denture tooth line was designed to offer additional features over traditional resin teeth ${ }^{(5)}$.

This study hypothesized that nano-hybrid composite teeth transmit more occlusal force to residual alveolar ridge than the acrylic teeth. Therefore, this study was conducted to compare the effect of different denture tooth material on mandibular residual ridge in completely edentulous patient using CBCT.

\section{MATERIAL AND METHODS}

Twelve healthy completely edentulous male patients their ages ranging between 50-65 years old who were seeking for the construction of complete maxillary and mandibular dentures were selected from the outpatient clinic of Prosthodontic Department, Faculty of Dentistry, Suez Canal University. Selected patients signed a consent form for participation after they were informed about the nature of the study.

The patients were selected according to the following criteria:

- The selected patients were free from any systemic disease that may negatively influence the rate of bone resorption.

- The edentulous ridges were covered with healthy firm and dense mucosa, i.e. free from signs of inflammation.

- Patients had no previous denture with nearly equal edentulous period.

All the patients received complete maxillary and mandibular dentures (which were constructed in the traditional way). According to the material of artificial teeth used, the patients randomly were divided into two equal groups each of which six patients: Group I: received complete maxillary and mandibular dentures with nano-hybrid composite teeth (SR Phonares II Typ, Ivoclar Vivadent, Liechtenstein) (Figure. 1). Group II: received complete maxillary and mandibular dentures with cross-linked acrylic teeth (Acry Rock, V code, Ruthinium group, Italy) (Figure. 2)

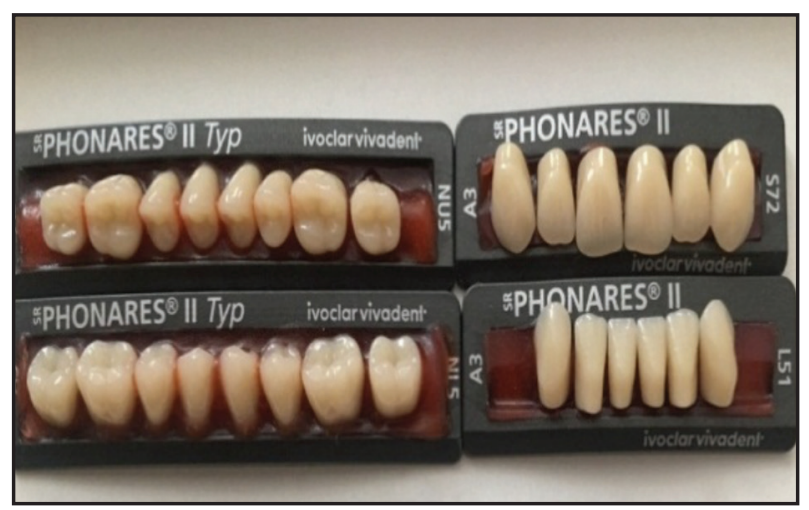

Fig. (1) Nano-hybrid composite resin teeth

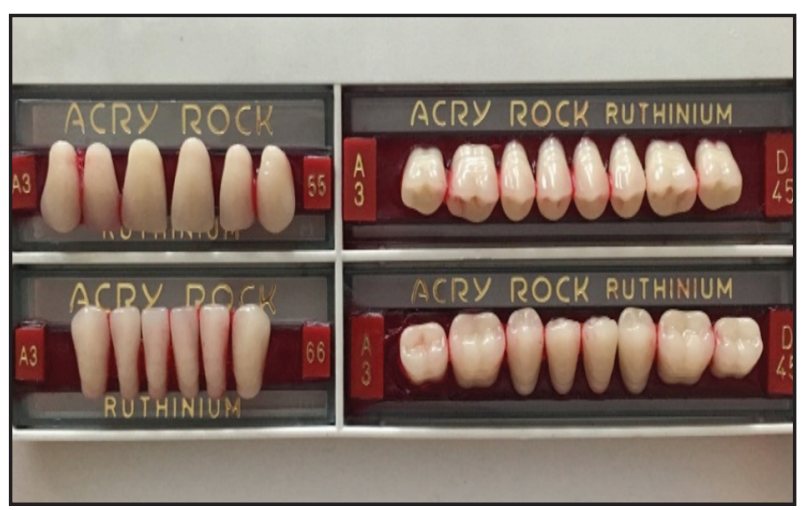

Fig. (2) Cross linked acrylic resin teeth

The evaluation was done radiographically using CBCT at the delivery, 6 months and 12 months follow-up appointments. The residual mandibular ridge was evaluated by assessing the height and width at consistent sites determined on the axial view: (midline, $15 \mathrm{~mm}$ ight and left to midline, 
distal border of right and left mental foramen, 10 $\mathrm{mm}$ distal to right and left mental foramen). The sites of measurement were determined on the axial view due to mandibular curvature for accuracy and repeatability (Figure. 3).

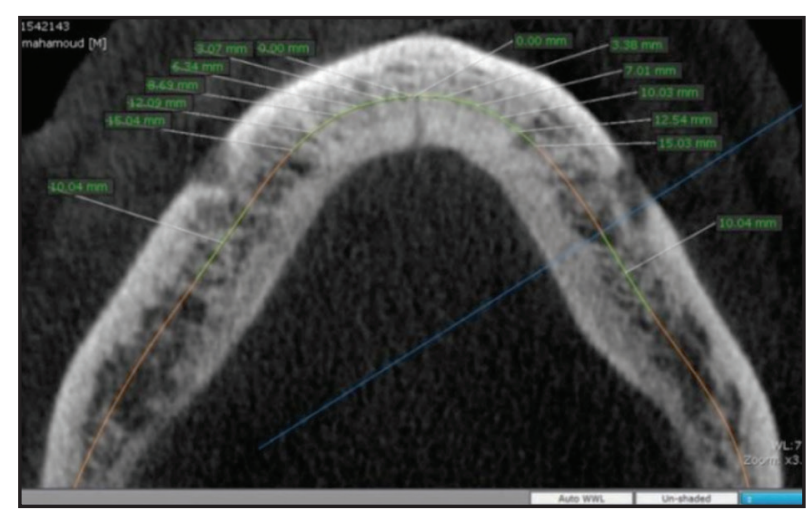

Fig. (3) Different sites of measurements on the axial view

The height of residual alveolar ridge was measured from the alveolar crest to the inferior border of the mandible on the reconstructed panoramic view. The anterior alveolar ridge height was measured at three fixed sites (midline, 15 $\mathrm{mm}$ right and left to midline) represented by the lines L, LR, LL respectively, while the posterior alveolar ridge height was measured by two vertical lines drawn on each side MR, ML (tangent to the distal border of the mental foramen) and PR, PL (10mm distal to MR, ML) respectively. These lines represent premolar and molar regions (Figure. 4).

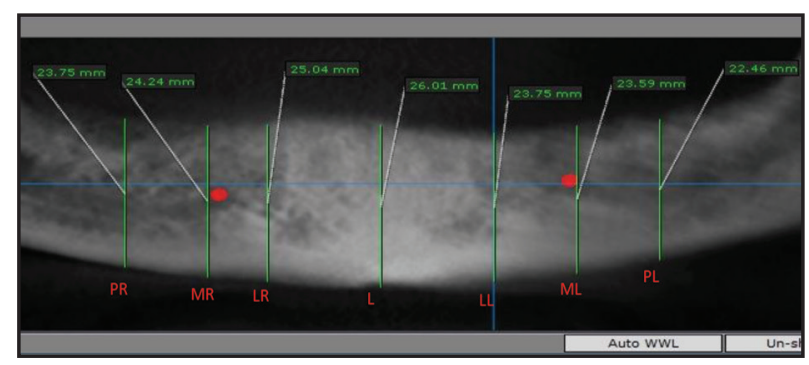

Fig. (4) Height measurement on the reconstructed panoramic view
Two measurements for the Width evaluation of residual alveolar ridge were performed on the crosssectional views of each previously mentioned site in either anterior or posterior mandible by extending two horizontal lines L1, L2 from exterior buccal to exterior lingual cortical plate. These two horizontal lines are parallel to each other and away from the alveolar crest $2 \mathrm{~mm}$ and $4 \mathrm{~mm}$ respectively. The distance from L1, L2 to the inferior border of the mandible were measured to be a reference in the following appointments (6 and 12 months) to ensure that width was measured at the same level each time (Figure. 5).

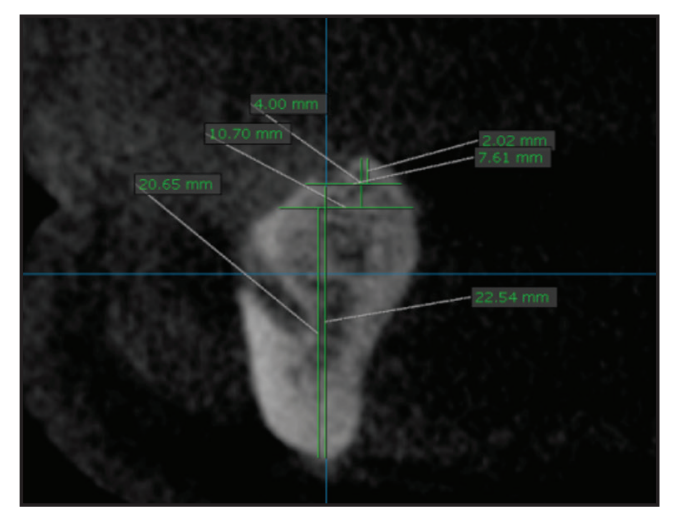

Fig. (5) Measurement of horizontal bone loss at delivery time $2 \mathrm{~mm}, 4 \mathrm{~mm}$ away from the alveolar crest

The measurements were done at the 3 observation period and the difference between readings was calculated and collected to obtain the mean of change in the alveolar bone height and width through the follow-up periods representing the crestal and horizontal bone loss respectively either in anterior or posterior regions.

Analysis of the CBCT radiographs was performed using special software program (OnDemand3DApp1.0.10.5385). Mean dimensional change of residual alveolar ridge height and width 
were recorded for each subject at different mandibular anatomical sites either for anterior or posterior mandibular regions

\section{Statistical analysis}

The data were collected, tabulated and statistically analyzed using (two-tailed t-test) as means, \pm standard deviation $(\mathrm{SD})$ and $P$ - value which is considered significant at $P \leq 0.05$ level and highly significant at $\leq 0.01$ level.

\section{RESULTS}

As shown in (Table 1) and (Figure. 6, 7), continuous bone loss in the height of the anterior and posterior region was observed for both groups during observation periods which is more noticeable in group I who received nano-hybrid composite resin teeth than group II who received cross-linked acrylic resin teeth. No statistically significant differences between two groups.

Table (1) Comparison of mean of the radiographic height changes in $(\mathrm{mm})$ of the anterior and posterior mandibular region between the two groups at observation periods

\begin{tabular}{|c|c|cc|c|c|c|c|c|c|c|}
\cline { 3 - 11 } \multicolumn{2}{|c|}{} & \multicolumn{3}{|c|}{ 0-6 month } & \multicolumn{3}{c|}{ 6-12 month } & \multicolumn{3}{c|}{ 0-12 month } \\
\hline hieght & material & Mean $(\mathrm{mm})$ & SD & p-value & Mean $(\mathrm{mm})$ & SD & p-value & Mean (mm & SD & p-value \\
\hline \multirow{2}{*}{ anterior } & composite & 0.570 & 0.678 & \multirow{2}{*}{0.709} & 0.544 & 0.235 & \multirow{2}{*}{0.875} & 1.114 & 0.832 & \multirow{2}{*}{0.745} \\
& acrylic & 0.488 & 0.323 & & 0.513 & 0.439 & & 1.001 & 0.727 & \\
\hline \multirow{2}{*}{ posterior } & composite & 0.730 & 0.682 & \multirow{2}{*}{0.491} & 0.565 & 0.318 & \multirow{2}{*}{0.988} & 1.295 & 0.756 & \multirow{2}{*}{0.612} \\
& acrylic & 0.535 & 0.486 & & 0.545 & 0.501 & & 1.080 & 0.885 & \\
\hline
\end{tabular}

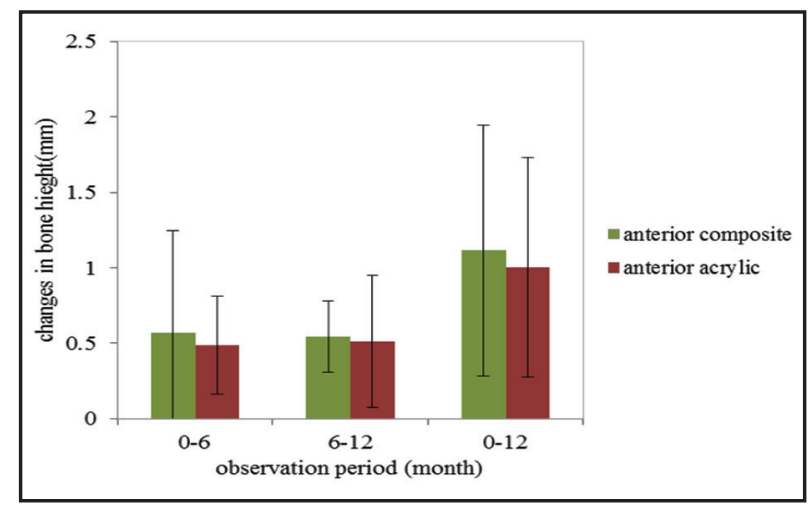

Fig. (6) Mean and standard deviation of changes in bone height in the anterior region of both groups at observation periods.

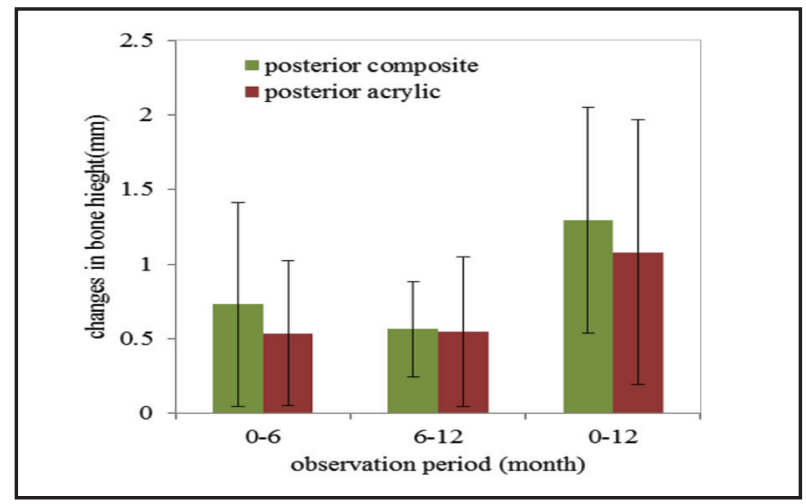

Fig. (7) Mean and standard deviation of changes in bone height in the posterior region between both groups at observation periods 
From (Table 2) and (Figure. 8, 9), higher rate of width reduction either in anterior or posterior mandibular regions was also in favor to group I than group II. An exception, at the first observation period (0-6 months), either for anterior or posterior regions, nearly no apparent difference in the bone width changes between the two groups

Statistically there was non-significant difference in the mean reduction of alveolar bone width between two groups at all observation periods in the anterior and even in the posterior region.

Table (2): Mean of the width changes in the anterior and posterior region of the mandible between the two groups at observation periods

\begin{tabular}{|c|c|cc|c|c|c|c|c|c|c|}
\cline { 3 - 11 } \multicolumn{2}{|c|}{} & \multicolumn{3}{|c|}{ 0-6 month } & \multicolumn{3}{c|}{ 6-12 month } & \multicolumn{3}{c|}{0 -12 month } \\
\hline width & & Mean (mm) & SD & p-value & Mean (mm) & SD & p-value & Mean (mm & SD & p-value \\
\hline \multirow{2}{*}{ anterior } & composite & 0.574 & 0.445 & \multirow{2}{*}{0.852} & 0.689 & 0.586 & \multirow{2}{*}{0.416} & 1.263 & 0.958 & \multirow{2}{*}{0.564} \\
& acrylic & 0.542 & 0.247 & & 0.504 & 0.309 & & 1.047 & 0.547 & \\
\hline \multirow{2}{*}{ posterior } & composite & 0.835 & 1.294 & \multirow{2}{*}{0.782} & 0.675 & 0.702 & \multirow{2}{*}{0.304} & 1.510 & 1.987 & \multirow{2}{*}{0.582} \\
& acrylic & 0.823 & 0.447 & & 0.444 & 0.292 & & 1.168 & 0.726 & \\
\hline
\end{tabular}

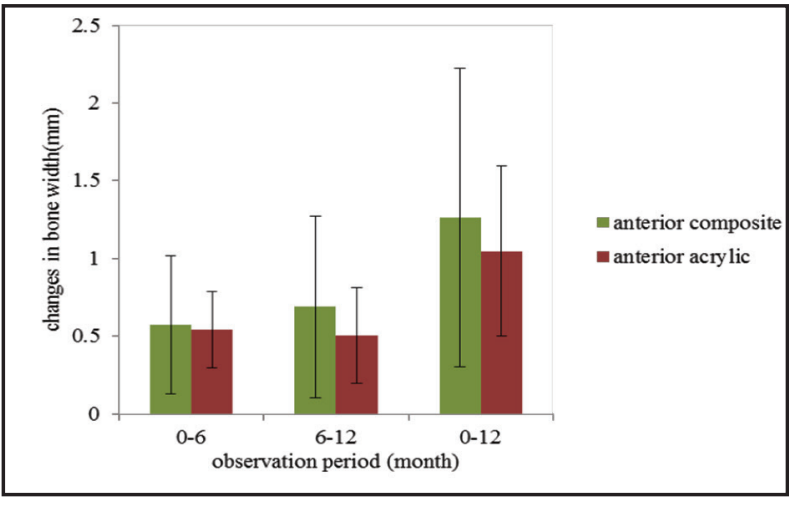

Fig. (8) Mean and standard deviation of changes in bone width in the anterior region between both groups at observation periods.

\section{DISCUSSION}

Male patients were selected in this study as the elderly females being in the postmenopausal phase and the estrogen hormone deficiency accelerates the bone loss in them leading to rapid ridge resorption especially in the mandible ${ }^{(6,7)}$.

The selected patients were free from any systemic disease that may interfere with normal

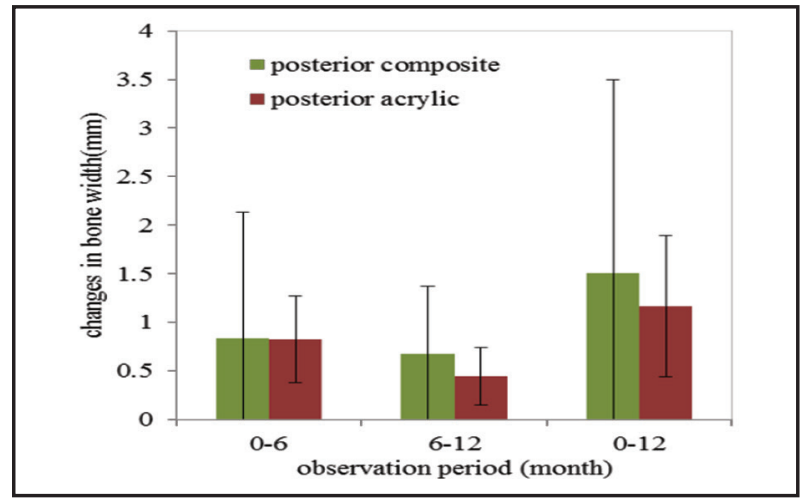

Fig. (9) Mean and standard deviation of changes in bone width in the posterior region between both groups at observation periods

bone metabolism such as diabetes mellitus and hyperthyroidism as these diseases stimulate the alveolar resorption process and the development of osteoporosis. In fact, any disturbance of the normal metabolic processes may lower the upper limit of mucosal tolerance and initiate inflammation ${ }^{(8,9)}$.

The edentulous ridge were Covered with healthy firm and dense mucosa as the oral mucosa beneath 
the denture plays a critical role in distributing occlusal loads to the underlying bony ridge over a large denture-supporting tissue interface ${ }^{(10)}$.

Nano composite resin (NCR) teeth seemed to be more suiTable for complete and partial dentures because of their good wear resistance. In addition, they consist of a newly designed nano-hybrid composite material, whose resistance to discoloration is significantly increased over that of conventional composite materials ${ }^{(11)}$.

Measuring height and width changes was performed as the vertical height and width of jaw bones are important factors for planning and success of the complete denture, as well as reduction of the residual ridge is one of the most important factors affecting denture support, retention, stability and masticatory function ${ }^{(12)}$.

The selected Seven measurements in the mandible at different anatomical reference points to obtain a fixed starting and ending points for measurement in each follow up appointment to ensure accurate data recording ${ }^{(13)}$.

Likewise mental foramina were considered a permanent point of reference, no matter how much resorbed the ridge is. It is situated in premolar areas, so act as point of transition from anterior to posterior areas. So it's a much easier, convenient and reliable method of assessing ridge resorption ${ }^{(14)}$.

In this study, patients were evaluated using CBCT imaging techniques as dental CBCT can be recommended as a dose-sparing technique compared with standard medical CT scans for common oral and maxillofacial radiographic imaging tasks, especially for hard-tissue studies and representations. From the radiation point of view, CBCT examinations can be used instead of CT to evaluate jaw morphology and prosthetic needs as a 3D image ${ }^{(15)}$.
The data obtained from the present study showed a decrease in the mandibular residual alveolar height and width with less reduction observed in group II which received dentures with cross-linked acrylic resin teeth. This may be due to their dampening effect as they have a certain degree of resiliency which provides energy absorption, so that acrylic resin teeth have higher shock-absorbing ability ${ }^{(16,17)}$.

The results of this study come in agree with the study of maximum pressure transmission which was observed with ceramic, nanocomposite, and acrylic denture teeth, respectively, and it was found that higher pressure values associated with ceramic denture teeth followed by nanocomposite and finally acrylic denture teeth ${ }^{(18)}$.

The results of this study showed that there was no statistically significant difference in the rate of alveolar ridge resorption between two groups either in height or width at all points of measurements. This may be due to the limitation of this study as the small sample size. To achieve a statistical power of $80 \%$, this study was completed with 12 participants.

\section{CONCLUSION}

Within the limitations of this study, the following conclusions are drawn: Despite of introduction of new denture tooth material as nano-hybrid composite resin teeth, the cross-linked acrylic resin teeth still considered the gold standard denture teeth regarding the degree of occlusal force transmission and preservation of residual alveolar ridge.

It is recommended that for the preservation of the residual alveolar ridge the acrylic resin teeth are the material of choice for complete denture construction, especially when comparing the cost difference between the two groups tested in this study, as the nano-hybrid composite teeth are very expensive. 
Certain recommendations regarding increasing sample size and length of observation period, for future researches about the effect of tooth material on the residual alveolar ridge.

\section{REFERENCES}

1. Singhal SH, Chand P, Singh BP, Singh SV, Rao J, Shankar $\mathrm{R}$. The effect of osteoporosis on residual ridge resorption and masticatory performance in denture wearers. Gerodontology 012;29:1059-1066.

2. Mosharraf R, Ziaei F, and Abbasi M. Effect of Denture Tooth Material on Load Transmission under Denture Bases. J Dent (Tehran) 2018;15:325-331.

3. Phunthikaphadr T, Takahashi H, Arksornnukit M. Pressure transmission and distribution under impact load using artificial denture teeth made of different materials. J Prosthet Dent 2009; 102:319-327.

4. Suwannaroop P, Chaijareenont P, Koottathape N, Takahashi H , Arksornnukit M. In vitro wear resistance, hardness and elastic modulus of artificial denture teeth. Dent Mater J 2011;30:461-468.

5. Preis V, Hahnel S, Behr M , Rosentritt M. Contact wear of artificial denture teeth. J Prosthodont Res 2018;62: 252-257.

6. Cooper LF. The current and future treatment of edentulism. J Prosthodont 2009;18:116-122.

7. Panchbhai AS. Quantitative estimation of vertical heights of maxillary and mandibular jawbones in elderly dentate and edentulous subjects. Spec Care Dent 2013;33:62-69.

8. Kumar L. Biomechanics and clinical implications of complete edentulous state. J Clin Gerontol Geriatr 2014;5: 101-104.

9. Jeffcoat M. The association between osteoporosis and oral bone loss. J Periodontol 2005;76:2125-2132.
10. Ahmad R, Abu-Hassan MI, Li Q, Swain MV. Three dimensional quantification of mandibular bone remodelling using standard tessellation language registration based superimposition. Clin Oral Implants Res 2013;24:1273-1279.

11. Ilangkumaran R, Srinivasan J, Baburajan K, Balaji N. Two Body Wear of Newly Introduced Nanocomposite Teeth and Cross Linked Four Layered Acrylic Teeth: a Comparitive In Vitro Study. J Indian Prosthodont Soc 2014;14:126-131.

12. Güler AU, Sumer M, Sumer P, Biçer I. The evaluation of vertical heights of maxillary and mandibular bones and the location of anatomic landmarks in panoramic radiographs of edentulous patients for implant dentistry. J Oral Rehabil 2005;32:741-746.

13. Tadiparthi DJ, Sujatha D. Tadiparthi J , Sujatha D. Evaluation of vertical bone heights of maxillary and mandibular residual ridges among edentulous diabetics by digital orthopantomograph. Int J Curr Res 2016;8:4060840612 .

14. Dany SS. Ahmed R, Kaul V, Kaul R, Dany SS , Khateeb SU. Analysis of severity of ridge resorption in West Uttar Pradesh population. IP Ann Prosthodont Restor Dent 2017;3:123-126.

15. Ozan O, Orhan K, Aksoy S, Icen M, Bilecenoglu B, Sakul BU. The effect of removable partial dentures on alveolar bone resorption: a retrospective study with cone-beam computed tomography. J Prosthodont 2013;22:42-48.

16. Kawano F, Ohguri T, Ichikawa T, Mizuno I , Hasegawa A. Shock absorbability and hardness of commercially available denture teeth. Int J Prosthodont 2002;15:243-247.

17. Mercier P, Bellavance F. Effect of artificial tooth material on mandibular residual ridge resorption. J Can Dent Assoc 2002;68:346-350.

18. Phunthikaphadr T, Takahashi H, Arksornnukit M. Pressure transmission and distribution under impact load using artificial denture teeth made of different materials. J Prosthet Dent 2009;102:319-327. 